\title{
Jóvenes centroamericanos: Una lectura desde los medios de comunicación escrita
}

Recibido: 19 de noviembre 2018

Revisado: 24 de enero 2019 Aprobado: 20 de agosto 2019

Bryan Roberto Vargas Vargas

Costarricense. Máster en Diseño Urbano de la Universidad de Costa Rica, sociólogo de la Universidad

Nacional de Costa Rica y arquitecto-urbanista. Investigador social del Programa Agenda Joven de

la Universidad Estatal a

Distancia de Costa Rica desde el 2012. Actualmente, estudiante en la especialización Liderazgo, cambio climático y ciudades de FLACSO Ecuador.

Intereses de investigación: juventud, ciudad, planificación urbana y territorial.

Correo electrónico: bvargas@uned.ac.cr

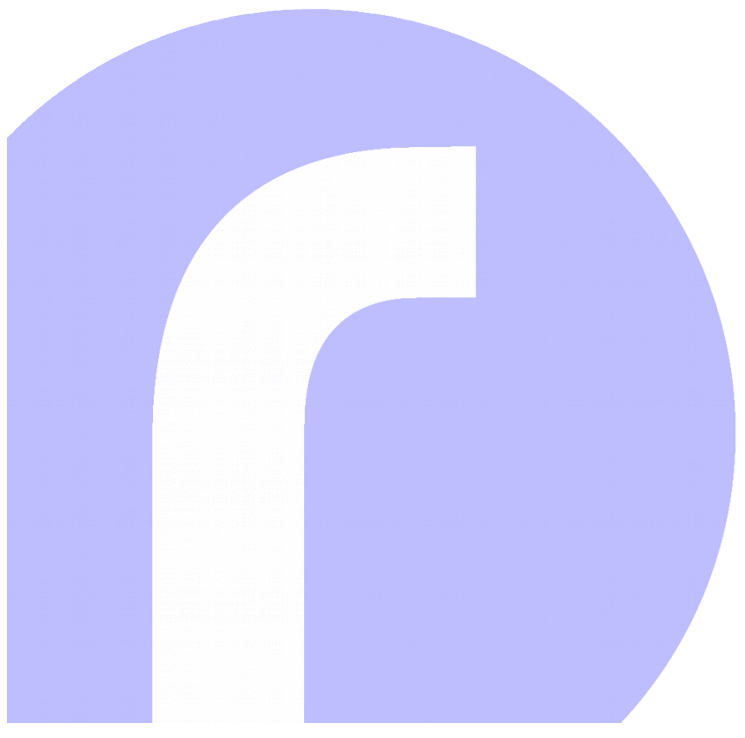

https://revistas.uned.ac.cr/index.php/rupturas Rev. Rupturas 10(1), Costa Rica, Ene-Jun 2020. ISSN 2215-2466. pp 1-23.
Resumen: El propósito del presente artículo es mostrar los espacios sociales, roles sociales y temáticas en los que se menciona a las personas jóvenes centroamericanas en la prensa escrita entre los años de 2013 al 2017. El trabajo se enmarca en los estudios de juventud que relacionan las condiciones sociales con las prácticas de quienes están en ese periodo de vida. Se exponen los datos estadísticos de un compilado total de 612 noticias centroamericanas, recopiladas de forma retrospectiva desde el año 2017 hasta el 2013 en las plataformas digitales de cada medio. Se busca profundizar teórica e históricamente en los dos temas en los que más se mencionó a las personas jóvenes como lo fueron la educación y la violencia.

Palabras clave: joven; estudiante; prensa de información diaria; flujo de noticias; proceso de comunicación; joven desfavorecido

\section{Young Central Americans: A Reading from the Written Media}

Abstract: This study aims to describe the social spaces, social roles and topics in which young Central Americans are mentioned in written mass media press between the years 2013 and 2017. Furthermore, this research belongs to youth studies that relate social conditions with the practices of those who are in that period of life. The retrospectively analyzed data derives from a 612 Central American news compilation from 2017 to 2013 of each online media's platforms selected. The two main topics in which young people are mentioned are theoretically and historically discussed in depth, namely violence and education.

Key words: youth; students; press news flow; communication process; disadvantaged youth 


\section{Introducción}

El presente artículo se enmarca en un proceso de investigación del Centro Agenda Joven de la Universidad Estatal a Distancia de Costa Rica, en el cual se exponen las reflexiones sobre la base de datos llamada "Jóvenes centroamericanos en prensa escrita", propiamente dando respuesta a la interrogante: ¿qué dicen los medios sobre los jóvenes centroamericanos? Esta base documental, igualmente elaborada por Agenda Joven, está compuesta por un total de 612 noticias centroamericanas compiladas durante el año 2017 y que, en su titular o desarrollo, reseñan a las personas jóvenes.

De las aristas consideradas para el análisis de la información, existe la conciencia de que se está trabajando con material periodístico en su totalidad, es decir, son los medios los que construyen información sobre las personas jóvenes, no son los jóvenes quienes relatan sus noticias o realidades. Por tanto, hablamos de un mensaje construido por parte de periodistas y medios, los cuales pautan los contenidos y acentuaciones que consideran pertinentes.

Además de mostrar los procesos y espacios sociales, se enfatiza en la separación sobre las personas jóvenes, por un lado, quienes cumplen con los papeles socialmente deseados y los otros que no lo logran y pueden ser asociados con violencia, en riesgo o personificando el riesgo del orden establecido.

El documento se referirá, en un primer momento, sobre el proceso metodológico cursado en la investigación, seguido de un desarrollo segmentado en apartados, en el cual se ubican las reflexiones teóricas, del contexto centroamericano y los datos estadísticos de la investigación, por último, las conclusiones de este.

1. La distribución temporal del número de noticias se presentó de la siguiente forma: en el año 2013 hubo un total de 126 noticias, durante el año 2014 se presentaron un total de 37 , en el año 2015 un total de 45 , durante el 2016 se registraron 382 y durante el 2017 un total de 21.

\section{Metodología}

El presente artículo tiene un alcance correlacional, en el que se representan frecuencias y asociaciones estadísticas de temas y espacios sociales sobre los cuales la prensa hace mención de las personas jóvenes, además se utilizan de forma cualitativa algunas noticias para ilustrar y profundizar en alguno de los temas o en el contexto.

Como se menciona en el preámbulo, la reflexión se enmarca en el estudio sobre juventudes desde el Centro Agenda Joven en Derechos y Ciudadanía, entidad que construyó un compilado de noticias centroamericanas entre 2013 y 2017. Dicha búsqueda documental se realizó vía web, utilizando los criterios "jóvenes", "personas jóvenes", “joven", "juvenil", "juventud”, “juventudes" en cada uno de los sitios oficiales de prensa, con el límite temporal de los años antes indicados ${ }^{1}$. 
Una vez identificados los contenidos se procedía a completar un instrumento ${ }^{2}$ de investigación digitalizado mediante Google Forms, que contenía los siguientes ítems: a) Fecha de identificación de la noticia, b) ID o número de entrada, c) Fecha de la noticia, d) Nombre del medio, e) Título de la noticia, f) URL de la noticia, g) Resumen de la noticia, h) Sección del diario, i) País, j) Localidad (la nota habla de su propio país o se refiere a otros países), k) Tema de noticia, I) Escritor de la noticia, m) Rol de la persona joven.

Sobre los tres últimos ítems, k) Tema, l) Escritor de la noticia y m) Rol de la persona joven, responden a criterios teóricos previamente estudiados; para el Tema, se construye una serie de respuestas cerradas sobre áreas ya trabajadas desde la ciencias sociales referentes a la población joven, estas se discutirán de forma más amplia en el desarrollo del artículo; en el caso del Escritor de la noticia, importaba determinar las fuentes, es decir, si es una presentación de hechos narrada por los periodistas, por algún experto, por alguna institución, opinión, o bien desde un joven o jóvenes.

Por último, el Rol de las personas jóvenes responde a ciertas preconstrucciones sobre la "juventud", como ser una población en riesgo, deportistas, consumistas, estudiantes, entre otras. Sobre los tres se ahondará en la discusión.

Para la selección de los periódicos por compilar, se priorizaron aquellos a los cuales el Estado Región (2013) ha dado seguimiento sistemático por su importancia en cada país, determinados en su investigación "Acciones colectivas en Centroamérica", pero principalmente porque fuera viable que tuvieran presentación tanto física como digital y que fuesen de publicación diaria. Finalmente, se obtienen los siguientes porcentajes:

Panamá América 21.3 \% (Panamá), El nuevo Diario 20.6 \% (Nicaragua), Prensa Gráfica 14.9 \% (El Salvador), La Nación 12.8 \% (Costa Rica), Diario El Heraldo $10.5 \%$ (Honduras), Diario Co Latino $6.4 \%$ (El Salvador), El Siglo $4.1 \%$ (Guatemala), El periódico 1.6 \% (Guatemala), Diario Extra 1.1 \% (Costa Rica). En el caso de Costa Rica, se consideró estratégico y viable revisar CR Hoy 6.7 \% (Costa Rica) por el alcance logrado por este medio digital en años recientes ${ }^{3}$.

Por otro lado, la etapa correlacional del presenta artículo consiste en una prueba de hipótesis bivariada en la cual se utilizan estadísticos como el $\mathrm{CHI}$ cuadrado y el PHI para determinar la intensidad de la asociación entre los temas noticiosos en cada uno de los países en análisis.
2. Es importante destacar que el proceso de compilación lo efectuaron estudiantes de la Universidad Estatal a Distancia de Costa Rica, quienes, capacitados en Agenda Joven, cumplen así horas de trabajo y obtienen a su vez una formación práctica en investigación.
3. Seguidamente, a modo de resumen se obtienen los siguientes números de noticias por país, el mayor dato se alcanzó en El Salvador con una frecuencia de 132 noticias, seguido de Costa Rica con 129 noticias, Panamá con 128 , Nicaragua con 124, Honduras con 64 y, por último, en Guatemala con 35 notas. 


\section{¿Qué dicen los medios sobre los jóvenes centroamericanos?}

\section{Apuntes sobre el estudio de la juventud y el contexto centroamericano}

Para iniciar interesa precisar dos ideas, la primera es la perspectiva sobre la juventud desde la que se posiciona la investigación y segundo, contextualizar la realidad centroamericana que se presenta para las personas jóvenes.

Pérez Islas (2008, 11-12) afirma que la década de 1920 es el momento en el que se problematiza a la juventud e inician las indagaciones sistemáticas en dos tendencias: por un lado, el culturalismo estadounidense al que le interesaba la influencia de los contextos culturales en la edad y, por otro lado, una postura sociológica, en dos aristas, la generacional funcionalista y la clasista (clase social).

En América Latina y el Caribe, Zúñiga $(2010,17)$ confirma esta tendencia culturalista que deviene de la Escuela Birmingham en autores como Reguillo, Feixa y Barbero, entre otros. Además, señala otras dos perspectivas, la primera es la corriente psicologista que considera al joven como no adulto o en transición a ser adulto y la segunda desde estudios cuantitativos o "cepalinos" que consideran a la juventud como número demográfico y su potencial productivo.

En el contexto latinoamericano, apunta Núñez $(2015,36)$ que, desde el primer estado del arte elaborado por Cecilia Braslavsky en 1989, sobresalen temáticas como participación, salud, trabajo, ocio, jóvenes diferentes (estudiantes, desocupados, mujeres). El mismo Núñez, en concordancia con José Antonio Pérez Islas, apuntan la consolidación de los estudios de juventud después del 2000 en una mezcolanza entre los temas de investigación y la definición de problemas sociales.

Sobre el mismo eje, Núñez (2015, 44-54) señala cuatro grandes temas latinoamericanos referentes a la juventud, como lo son: a) la relación escuela y el mundo del trabajo, b) culturas juveniles, c) políticas públicas y d) violencia en dos dimensiones (criminalización/seguridad y conflicto/exclusión social). Muchos de estos temas se hacen transversales y es entendible en el contexto de condiciones sostenidas de desempleo, conflictos armados, desigualdad y exclusión educativa, como mostraremos más adelante.

Agrega Núñez que este aumento de población fuera de la escuela y del mundo del trabajo tiende a ser "contenido noticioso" y, como señala Centeno (2014), hay una penalización de la pobreza a través de las notas rojas y los medios de comunicación, donde las personas pobres (excluidas de la educación y el empleo) son las protagonistas de riñas callejeras, episodios de violencia y robo, y actividad delictiva de pandillas juveniles, por lo que algunos estudios versan también acerca de resaltar la heterogeneidad de las juventudes.

Lo importante de los anteriores temas es que trazan áreas donde la sociedad percibe como problema a los jóvenes o que les aquejan. Este señalamiento es importante, ya que hace una ruptura acerca de que son los y las jóvenes, 
dejando de lado el enfoque biológico o etario y apelando, entonces, a la condición social y los modos de vivencia.

En buena medida, el presente artículo insiste en esta postura analítica que relaciona a las juventudes centroamericanas con sus condiciones sociales y los ámbitos sociales en los que los enmarca la prensa, muy encuadrado en lo que Zúñiga $(2010,20)$ Ilama juventud como "adjetivo", que califica personas, procesos sociales y espacios sociales como la principal premisa analítica.

Por otro lado, la autora Patricia Vega (2016), en su estudio de las reflexiones académicas sobre los medios de comunicación en Centroamérica entre los años de 1980-2015, apunta el interés académico en lo convulsa y cambiante de nuestra región, definiendo tejidos que han sido propios de toda la región y, por tanto, noticiosos en todos.

Indica Vega (2016) que durante el periodo de estudio (1980-2015), cuando de análisis académicos sobre los medios de comunicación se trata, algunos temas pueden ser constantes en todos los países, por ejemplo: a) las posiciones ideológicas ante la guerra fría, b) la importancia de la prensa en el posicionamiento de ideas ante los conflictos internos, c) la cobertura de los conflictos propios de cada país, d) los medios y su relación con el poder político o económico, e) el crimen organizado y f) los medios en la construcción de la democracia.

Según la autora, que lo anterior sea así responde a las realidades en común de los países como son los conflictos armados, los modelos económicos que no pudieron compensar las carencias materiales, los regímenes autoritarios, revoluciones populares, intervencionismos de los Estados Unidos y los procesos de paz. Condiciones y procesos compartidos que se concatenaron para formar Estados debilitados, en crisis económica y un aumento de la pobreza y la violencia (Vega 2016, 119-121).

En este contexto, uno de los sectores más afectados fue el de aquellos en edad joven, como lo veremos más adelante con los datos, aunque cada país con sus particularidades, pero de igual forma se confirma la tendencia de que las manifestaciones de violencia estén protagonizadas por jóvenes, por lo que los discursos sobre la criminalización joven no se hicieron esperar y la mano dura fue la respuesta política, señala Lemus $(2018,47)$ : "El núcleo central de los discursos de criminalización parte de que ciertas agrupaciones típicamente juveniles, las maras, son las principales responsables de la inseguridad ciudadana".

Apunta Mejía (2005) que la desarticulación de una persona miembro de una pandilla y la sociedad se profundiza en la escuela y el trabajo. "Las incapacidades de la institucionalidad de poder integrar a los jóvenes pueden transformar el sufrimiento familiar en un sentimiento de rechazo y conflicto con los niveles de autoridad en la sociedad." (Mejía, 2005, 394).

Como vemos, lo convulso de la región en temas relacionados con conflictos armados, carencias materiales y exclusión educativa y laboral fueron parte de los detonantes para el estudio la juventud de la región y el material noti- 
4. Los medios transmiten la representación social de que la vida está en riesgo; riesgo de ir por las calles, riesgo de conducir autos, riesgo al tener relaciones sexuales "sin protección", riesgo de ser mujer, riesgo de perder el trabajo, riesgo de ataques terroristas, y por supuesto toda otra serie de riesgos que difunden en la sociedad la idea de que hay una crisis permanente que afecta la vida cotidiana (Menéndez y Di Pardo 2008, 11). cioso. Nos muestra, además, la tendencia de ámbitos de investigación y de las realidades acerca de las cuales se discute sobre juventud, esto permite, entonces, establecer temas en que se ubican los jóvenes como lo son, sin duda, la escuela, el trabajo, y las formas de violencia.

\section{¿Cuál es laatención qe se debe tener ante la información mediática?}

Es necesario sumar en este sentido y vincularlo con la realidad centroamericana que hemos descrito, afirman Mayorga y del Valle $(2008,19)$ que los medios de comunicación son agentes mediadores de la realidad y actores en el proceso de socialización, mediante "experiencia mediática" que le permite al receptor experimentar hechos y conocer realidades que, en lo referente a su interacción social, jamás podría acceder, por lo tanto, los individuos aprenden de los discursos que los medios de comunicación construyen y, a su vez, configura su conocimiento social sobre determinado fenómeno (representaciones sociales).

Menéndez y Di Pardo (2008, 11), quienes retoman conclusiones de Bourdieu, Castells y otros, hacen una interpretación sobre el fenómeno mediático en su estudio sobre "La representación social negativa de los procesos de salud/ enfermedad/atención en la prensa escrita", estos autores señalan que los medios están ocupados en mostrar únicamente los hechos negativos de forma alarmista ${ }^{4}$, ya que parten de la premisa de que las buenas noticias no son noticia. Más adelante, veremos que en esta lógica encajan las poblaciones "jóvenes" cuando se asocian con ser una población en riesgo, desocupada, desinteresada, agresiva, entre otras. Sobre lo anterior, los autores indican:

Como venimos señalando, los temas de investigación sobre juventud responden en buena parte a la definición de los problemas sociales, dificultades que, a su vez, nutren de información que se presentará como noticia, esto nos lleva a prestar atención a los temas asociados a los jóvenes que posibilitan ese éxito noticioso y los contextos de cada uno de los países centroamericanos.

Para la primera década del siglo XXI, la región centroamericana presenta una realidad compleja y una violencia que la caracterizaba, en particular Honduras, Guatemala y El Salvador, donde se manifiestan altos indicadores en la tasa de homicidios, de los más altos del mundo para ese momento. El Salvador, el más alarmante de los tres presenta otra condición, pues, de estos casos, la victimización de la población "joven" en 2005 fue de un 92.3, superando a Guatemala con 55.4 en 2004 (Figueroa Ibarra con base en Waiselfisz 2011, 9).

Esta correlación o imaginario de joven como problema social se menciona porque ha sido planteado por autores como Aliaga Sáez y Escobar Monje (2006) y Duarte Quapper y Littin Menz (2002). Estos últimos afirman, en su estudio sobre "Niñas, niños y jóvenes: construyendo imágenes en la prensa 
escrita", que los mecanismos más utilizados en la producción de esta imagen del "joven problema" son dos:

i) niños, niñas y jóvenes como peligrosos para la sociedad, en la medida que no se adaptan a las normas sociales que ha definido el mundo adulto; y, ii) niños, niñas y jóvenes como infractores de la ley que busca regular la convivencia social (Duarte y Littin 2002, 65).

En todo caso, si retomamos la propuesta analítica juventud como "adjetivo", que califica personas, procesos sociales y espacios sociales, el procesamiento que se haga de un acto de violencia por parte de la prensa debe atenderse, dado que, por un lado, puede explicar la problemática de la agresión constante o mostrar siempre en asociación las personas jóvenes con crimen o justificando la mano dura que explicaba Lemus (2018) y así será recibido por los receptores de la información. Para reforzar la premisa, la siguiente tabla de temáticas obtenidas según país.

Tabla 1. Centroamérica: Porcentaje de temas noticiosos por país centroamericano, 2013 y 2017

\begin{tabular}{|c|c|c|c|c|c|c|c|c|}
\hline & & & & Paí & & & & \\
\hline & & $\begin{array}{l}\text { Costa } \\
\text { Rica }\end{array}$ & $\begin{array}{l}\text { El } \\
\text { Salvad } \\
\text { or }\end{array}$ & $\begin{array}{l}\text { Guate } \\
\text { mala }\end{array}$ & $\begin{array}{l}\text { Hondu } \\
\text { ras }\end{array}$ & $\begin{array}{l}\text { Nicara } \\
\text { gua }\end{array}$ & $\begin{array}{l}\text { Pana } \\
\text { má }\end{array}$ & Total \\
\hline & Violencia & 15.5 & 29.8 & 22.9 & 71.9 & 29.0 & 16.4 & 27.8 \\
\hline & Tecnología & 10.9 & .8 & 5.7 & & 4.8 & 3.1 & 4.4 \\
\hline & Salud o sexualidad & 13.2 & 3.1 & & 4.7 & 3.2 & 8.6 & 6.4 \\
\hline & Participación & 7.8 & 12.2 & 20.0 & 4.7 & 6.5 & 3.9 & 8.0 \\
\hline$\stackrel{\widetilde{\sigma}}{\varepsilon}$ & $\begin{array}{l}\text { Mundo del trabajo } \\
\text { o negocios }\end{array}$ & 10.1 & 8.4 & 20.0 & 1.6 & 6.5 & 14.1 & 9.5 \\
\hline & Institucionalización & 2.3 & 8.4 & 2.9 & & & 2.3 & 2.9 \\
\hline & Educación & 16.3 & 17.6 & 14.3 & 4.7 & 13.7 & 17.2 & 14.9 \\
\hline & Cultura y deporte & 18.6 & 16.0 & 5.7 & 3.1 & 21.0 & 22.7 & 17.0 \\
\hline & $\begin{array}{l}\text { Accidentes } \\
\text { (tránsito, laborales, } \\
\text { hogar, etc.) }\end{array}$ & 5.4 & 3.8 & 8.6 & 9.4 & 15.3 & 11.7 & 9.0 \\
\hline To & & 100.0 & 100.0 & 100.0 & 100.0 & 100.0 & 100.0 & 100.0 \\
\hline
\end{tabular}

Fuente: elaboración propia

La tabla anterior, elaborada desde la base de datos en cuestión, muestra que el tema más frecuente en el que se menciona a las personas jóvenes es en Violencia, mayormente en Honduras y El Salvador; seguido de los ámbitos de 
Educación y Cultura y Deporte. Correspondiendo la frecuencia del tema con los indicadores de violencia, siendo, fundamentalmente, la "experiencia mediática" que se desea implantar. Recordemos que la tendencia en la región para atender la creciente violencia fue la mano dura, por lo que el mensaje mediático toma importancia en el posicionamiento de determinada acción.

Lo anterior, teóricamente corresponde con las miradas institucionales con las que se ha comprendido a las poblaciones jóvenes, Pérez (2000) señala cuatro, a saber: a) la juventud como etapa transitoria, b) la juventud como futuro, c) la juventud como rol total y d) la juventud como "buenos o malos". En el caso de la mirada de los jóvenes como "buenos o malos", es una relación binaria donde los primeros son aquellos que cumplen los roles establecidos, ya sean trabajadores, estudiantes, deportistas, emprendedores, entre otras cualidades acordes con el orden social mientras que los malos son aquellos fuera de estos espacios, desertores del sistema educativo, vagos, desempleados, ninis o protagonistas de la violencia.

Esta lógica sobre los jóvenes en prensa escrita ya fue identificada también por Saintout (2013) en su estudio sobre los jóvenes en la Argentina, en el cual señala que existen los "Los exitosos: casi Ángeles" que responden a los modelos hegemónicos, el joven exitoso, aceptable y deseable para el modelo político económico neoliberal y por otro lado "Los peligrosos: los desangelados" los que no tiene nada que perder por decisión propia y, por tanto, son incontrolablemente peligrosos para el resto de la sociedad y se justifica la represión sobre estos (Saintout 2013, 52-53).

Como parte de esta contextualización centroamericana, salirse de esta comprensión binaria es difícil y la posibilidad concreta de permanecer en los espacios deseados no es sencilla, ni se da en igualdad de oportunidades, parte de esto se evidencia en las noticias mismas, sobre el mundo del trabajo, por ejemplo: La Nación publica "300.000 jóvenes del país trabajan o buscan trabajo" (Leitón 2013), señalando que el desempleo e informalidad son mayores en ese grupo y el ingreso menor, agrega que "el $50 \%$ de los que laboran lo hacen en comercio, agricultura e industria, según Censo 2011". CR Hoy (Rojas 2013) publica "Jóvenes ticos con más problemas de empleo en Centroamérica; recomiendan medidas para encontrar trabajo", exponiendo la dificultad para personas jóvenes de encontrar trabajo incluso con un título de educación superior o algún conocimiento técnico.

En algunas noticias, se consulta a expertos en el tema que dan una lectura distinta del fenómeno, por ejemplo, "Delincuencia aumenta porque jóvenes 5. René Quevedo, consultor en no encuentran empleo", según experto ${ }^{5}$ (Panamá América 2014), los jóvenes inserción laboral de 15 a 29 años representan el $65 \%$ de las personas que buscan empleo en Panamá, pero solo están obteniendo uno de cada 28 nuevos empleos generados, "lo que significa que es 20 veces más probable que un joven cometa un delito que un extranjero"; esta noticia, en particular, señala una propensión de los jóvenes por delinquir, pero se agrega que es resultado de un problema mayor de empleo generalizado. 
El Siglo, con fecha 12 de junio de 2016, presenta el titular "Desnutrición y violencia amenazan a niños y jóvenes" en el que se reseña la posición del ministro de Educación guatemalteco, quien expuso que los niños y adolescentes guatemaltecos enfrentan varias situaciones que se conocen como "riesgos permanentes", la desnutrición crónica que afecta a la mitad de este sector y que disminuye las capacidades, además de la integración de los niños en el crimen organizado donde muchos son utilizados por las pandillas para cometer delitos (El Siglo 2016).

El Siglo (Ruiz 2016) publica "Se estima que solo hay 35 mil puestos de trabajo", en el cual se explica que el bajo número de empleos que se ponen a la disposición de los jóvenes no alcanzan para el gran número que se gradúa cada año. Según la autora, el $12 \%$ de los doscientos mil jóvenes que ingresan al mercado logran un empleo formal; con este panorama, el $95 \%$ de las personas en posibilidad y necesidad de empleo han considerado emprender algún negocio, el resultado es que ocho de cada diez personas en Guatemala son trabajadores independientes y estos, al momento de iniciar sus finanzas, se encuentran con la dificultad de mantenerla en un tiempo mayor de 42 meses.

Esta dificultad de permanecer en ciertos espacios sociales son paradojas para los sujetos que practican ese momento de vida, por un lado, deben ser el futuro y reproducir la organización social ya establecida, innovar y no cometer los errores que ya otros han cometido, pero se espera cualquier cosa de los jóvenes; en todo caso, el contexto centroamericano descrito no ofrece garantía de algún futuro, son "esperanza bajo sospecha", como les ha llamado Rodríguez $(2000,27)$, pero entendibles también en el contexto que describe el mismo autor:

Las condiciones de exclusión social que afectan particularmente a las juventudes populares urbanas, se acompañan de un nivel de exposición inédito a propuestas masivas de consumo, y de una centralidad igualmente inédita de la cultura juvenil en la sociedad. Todo ello define una situación de anomia estructural, en la cual los jóvenes tienen una relativamente alta participación simbólica en la sociedad que modela sus aspiraciones, y una participación material que no permite la satisfacción de esas aspiraciones por cauces legítimos. La combinación de todos estos elementos contribuye a la formación de subculturas marginales, de pandillas y barras que tienen códigos propios, subculturas que suelen incorporar y consolidar en el 
tiempo, los hábitos y comportamientos que surgen como correlatos socialmente disruptivos de las situaciones de marginalidad y exclusión social (Rodríguez 2003, 4).

Ahora, los datos obtenidos permiten seguir discutiendo en la misma línea, las siguientes figuras muestran los resultados estadísticos y otros elementos en la reflexión.

\section{Los resultados obtenidos}

Para iniciar, la figura 1 presenta quién elaboró el texto; en este caso, el mayor porcentaje de las noticias es redacción del medio, ya sea por su equipo periodístico o editoriales. Muy por debajo están las redacciones en las que se le consulta a alguna institución, expertos sobre algún tema en específico o a los jóvenes involucrados.

Figura 1. Centroamérica: Porcentaje de redacción noticiosa en Centroamérica, 2013 y 2017

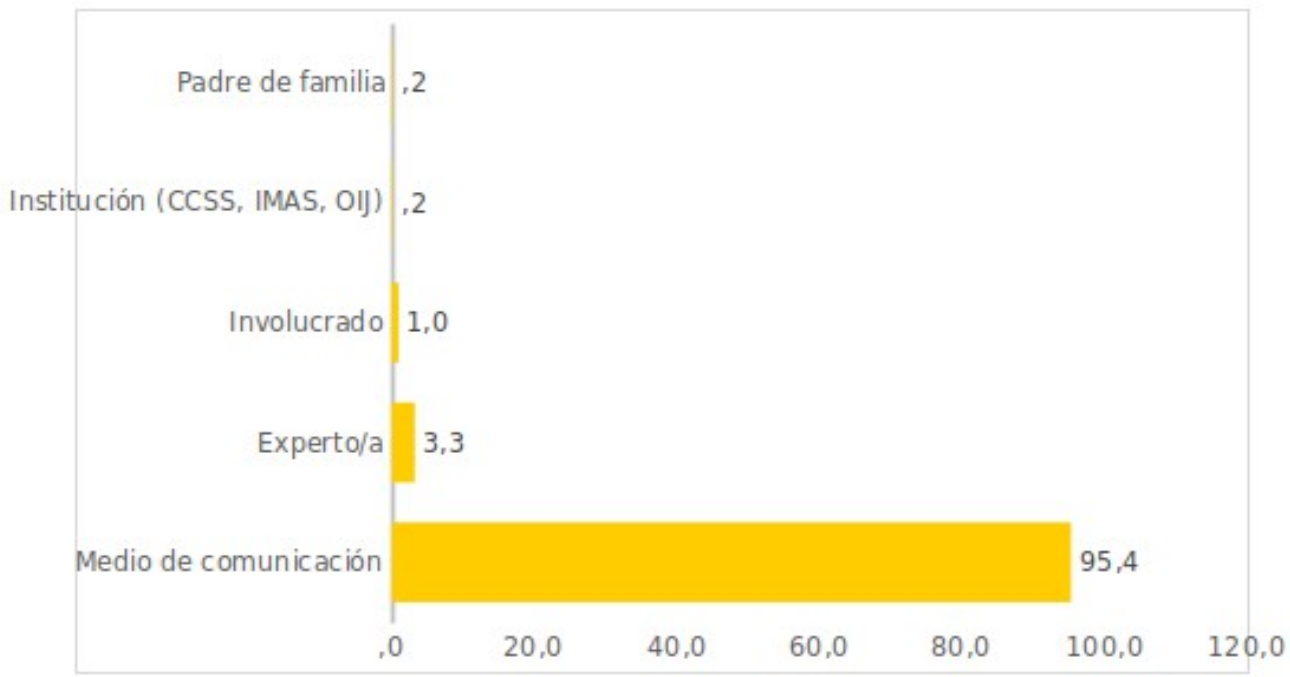

Fuente: Elaboración propia 2019

Esto es significativo, ya que, como se viene planteando, es una línea editorial dada que hace referencia a los jóvenes, por tanto, acentúa lo que es relevante al medio. La distribución del material noticioso, según la sección dentro del diario, principalmente se mostró en Nacionales con un $47 \%$, seguido del espacio de Sucesos con un $18 \%$ y en menor medida en los espacios de Opinión y Economía. 
Figura 2. Centroamérica: Porcentaje de roles juveniles en prensa escrita centroamericana, 2013 y 2017

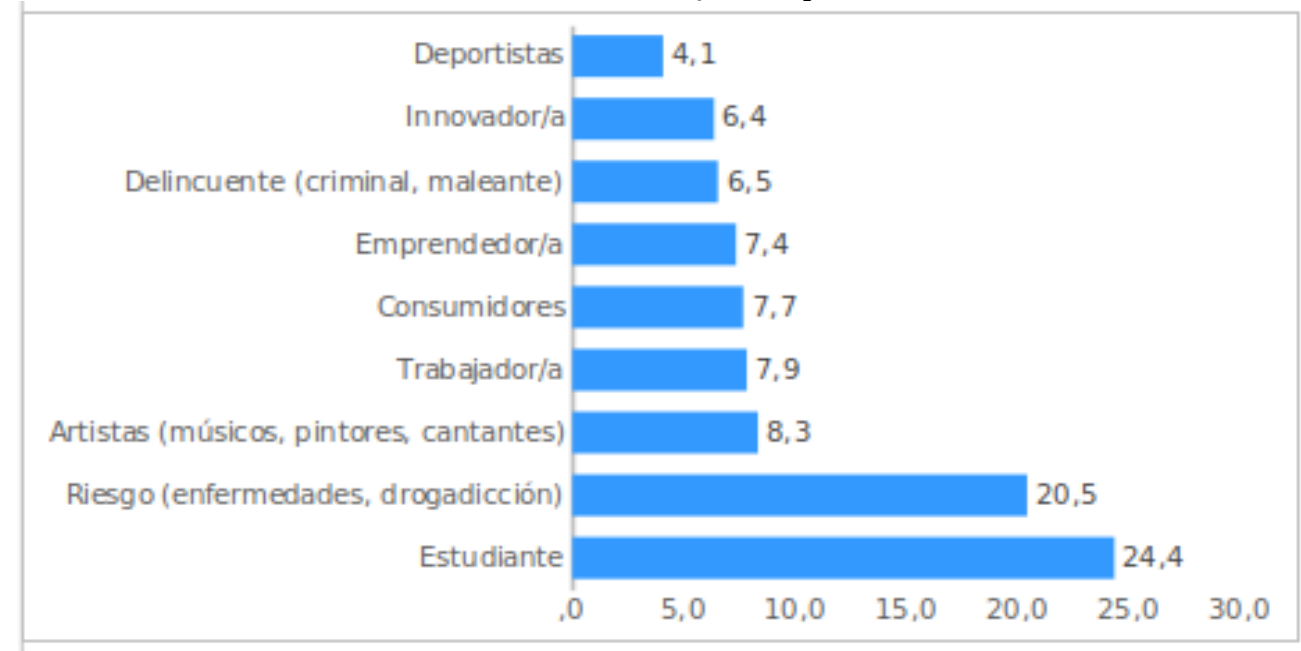

Fuente: Elaboración propia, 2019

La figura 2 muestra los roles que se le asignan a las personas jóvenes en el desarrollo noticioso, es decir, acciones o ámbitos de la vida social que se esperan o se imponen sobre este grupo. Los datos obtenidos nos muestran que el mayor rol asignando es en su accionar como estudiantes, seguido como una población en riesgo y tercero con roles artísticos. Es poco frecuente la asignación de roles como "nini" ${ }^{\circ}$ de alguna "subcultura", ambas se han utilizado para homologar a la población joven.

Ahora, la figura 3 muestra la distribución porcentual de los temas en los cuales se enmarcan a las personas jóvenes, el porcentaje más amplio es sobre Violencia con un $28 \%$ de las noticias, seguidos por Cultura y Deporte con un $17 \%$ y Educación con un $15 \%$. En menor medida, los temas tratados son tecnología con un $4 \%$ e Institucionalización (tiene que ver con la vinculación entre jóvenes y alguna institución gubernamental en específico) con un $3 \%$.

Desde Pérez (2000), tanto los roles como los temas dominantes encajan en el desdoblamiento de "buenos o malos", "estudiantes o en riesgo", respectivamente. Los "buenos" y "malos" jóvenes es un pareo desde el cual también se posiciona Reguillo $(2000,23)$ y señala que "existen a través del sistema político jurídico que les otorga un 'lugar' y les demanda unas prácticas; existen, a través del discurso que el mercado elabora sobre y para ellos".

Zúñiga $(2010,53)$ retoma premisas de Bourdieu indicando que "los esquemas que permiten performatividad son de carácter binario y modelan relaciones sociales, las instituciones y los cuerpos desde posiciones fundamentales como: adulto/joven, modelo/monstruo, bueno/malo". Quien se adapte a esta proyección, tiene la "condición de posibilidad" para ser "grande": es hombre, adulto, civilizado y modelo; quien posee las características opuestas, es susceptible de ser monstrificado o cooptado.
6. La discusión sobre "ninis", según Borunda Escobedo (2013), como propia de un cambio paradigmático iniciado en la segunda década de los años dos mil en que se elabora el constructo como categoría de análisis socioeconómico, pero que se le adhiere una connotación despectiva no prevista hacia las personas entre 15 y 30 años que no han podido acceder a las instituciones educativas y a los mercados laborales.

7. La idea del Rol total en Pérez Islas (2000). 
Figura 3. Centroamérica: porcentaje de temas noticiosos sobre personas jóvenes en Centroamérica, 2013 y 2017

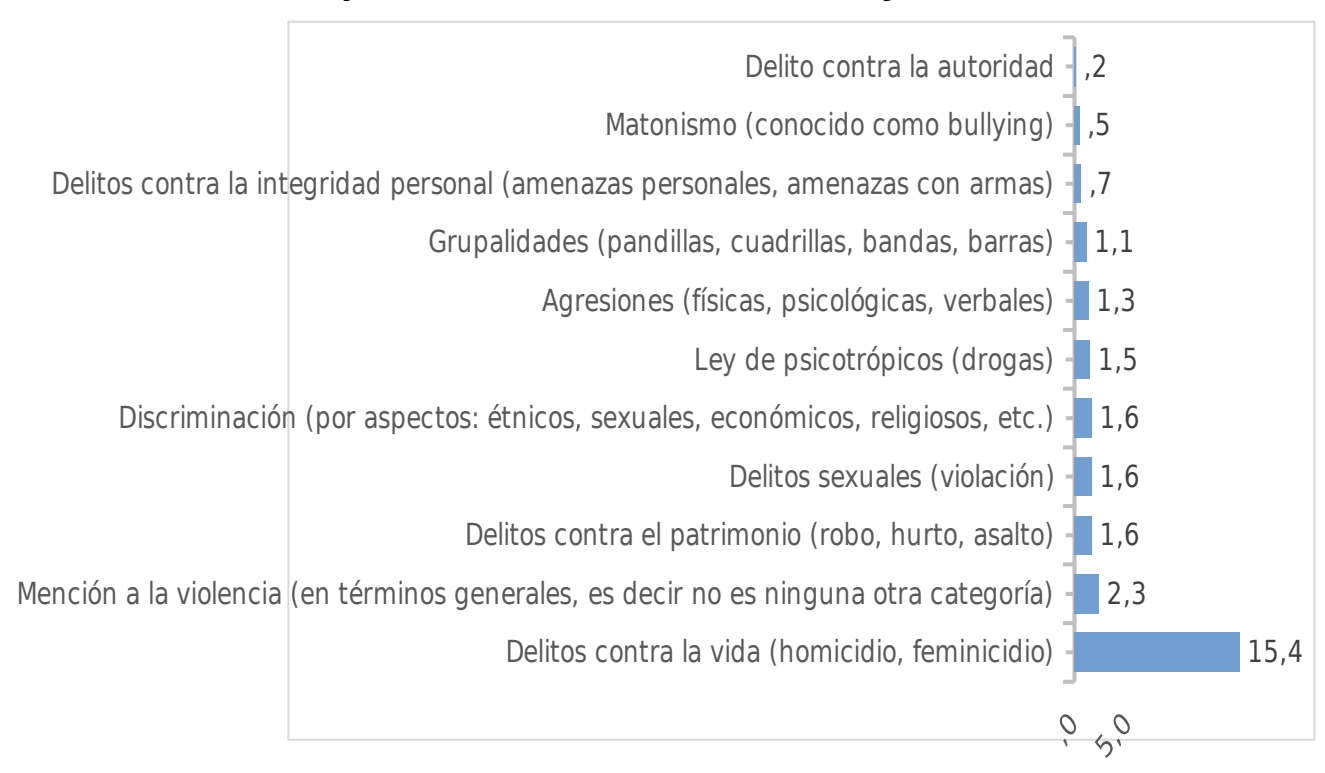

Fuente: Elaboración propia

8. Para el momento del compilado, no había iniciado el conflicto político en Nicaragua (2018), por lo que probablemente para este momento el panorama puede ser otro.
En un contexto convulso como el descrito en apartados anteriores, es consecuente que se manifieste también en la producción noticiosa; estos temas coinciden con lo señalado en el estado del arte de Núñez (2015) como frecuentes en el estudio de juventud en Latinoamérica, la frecuencia del tema de la violencia también tiene un respaldo empírico, señala Jiménez $(2016,169)$ :

La Organización Mundial de la Salud (OMS) señala que, cuando en un país la tasa de homicidios por cada 100 000 habitantes es mayor de 10 personas, en ese lugar se vive una verdadera problemática. A excepción de Costa Rica y Nicaragua ${ }^{8}$, el resto de las naciones centroamericanas experimenta tal problemática debido a que sobrepasan ese rango: Panamá (20-30), El Salvador (mayor de 30), Guatemala (mayor de 30) y Honduras (mayor de 30). 
Figura 4. Centroamérica: porcentaje de distribución de formas de violencia en prensa escrita Centroamérica, 2013 y 2017

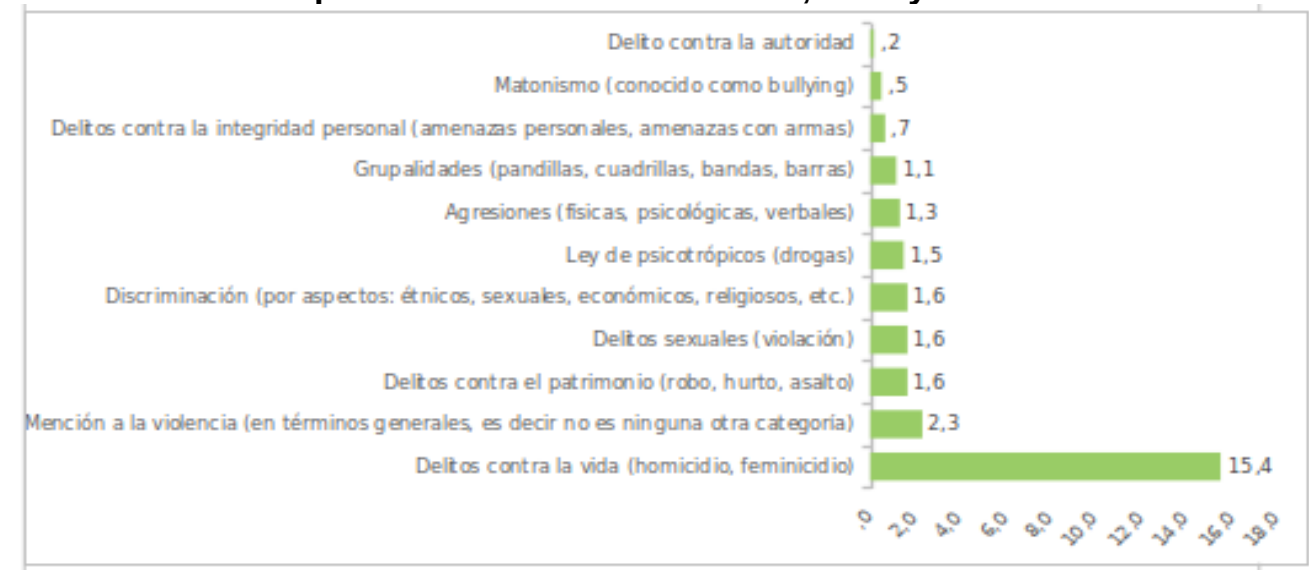

Fuente: Elaboración propia

La figura 4 muestra los subtemas que se desprenden del tema de Violencia, del mismo se puede determinar que el rubro más alto es Delitos contra la vida (entiéndase asesinatos o femicidios), muy por debajo de cualquier otra forma de violencia indicada, principalmente en comparación a los actos de Delitos contra la autoridad y Matonismo en las instituciones educativas.

De los restantes datos obtenidos, los subtemas que se desprenden del ítem del mundo del trabajo, el mayor de los rubros es el de Emprendimiento con un $28 \%$, seguido de los jóvenes en Empleo con un $27 \%$ y, posteriormente, jóvenes en Desempleo con un $21 \%$. En menor medida se habla sobre "nini" (ni estudian, ni trabajan) con un $9 \%$ e Informalidad laboral con un $2 \%$.

Por otro lado, los subtemas que se desprenden del tema de Cultura y Deporte, segundo tema en importancia, principalmente son en actividades relacionadas con Música (conciertos, festivales y otros) con un $24 \%$, seguido de Valores, es decir, actividades en que los jóvenes muestran civismo, respeto y otros con un $14 \%$.

No hubo frecuencia importante de noticias en temas de participación, ya sea en política, voluntariados, comités cantonales, asociaciones de desarrollo, juntas educativas, acciones colectivas, movimientos sociales, entre otros. Tampoco hubo continuidad en el tema de Salud, Sexualidad, Tecnología y Consumo, esto es un hallazgo en tanto se consideran ámbitos sociales que se asocian con la población en edad joven, pero no lo son en este compilado noticioso (ver tabla de contingencia 1 ).

Como datos fuertes, tenemos que los dos roles predominantes son: a) el estudiante y b) el sujeto en riesgo y los temas más frecuentes fueron los siguientes: 1) el ámbito educativo-recreativo y 2) los actos de violencia. Por otro lado, el problema del desempleo-empleo es transversal, al igual que emerge el emprendimiento como posibilidad individual de los y las jóvenes para crear sus propios recursos. 
Figura 5. Centroamérica: asociación estadística entre temas noticiosos por país, 2013 y 2017

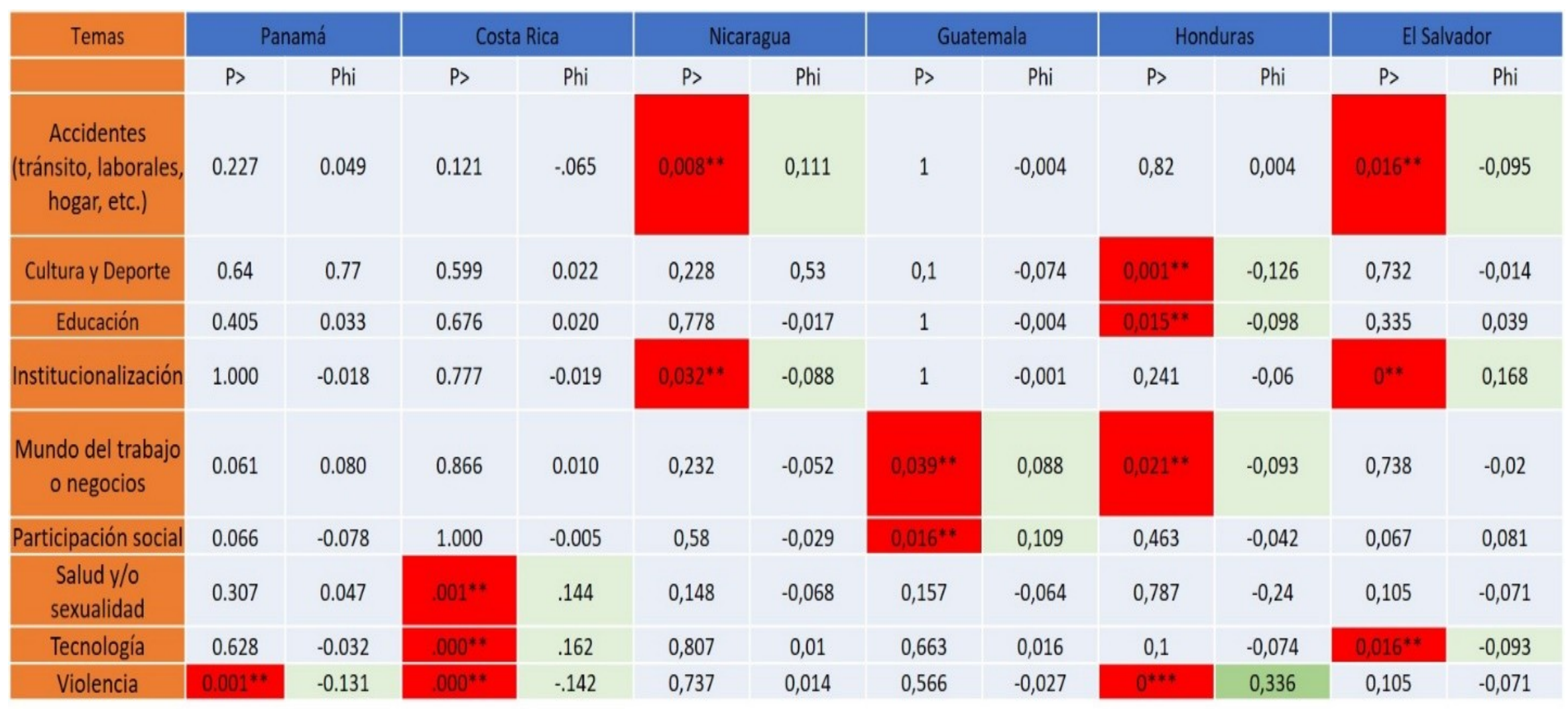

Fuente: Elaboración propia 
Si bien ya vimos que en conjunto hay predominancia de dos temas y dos roles; por las características de cada país, surge la hipótesis de existir asociación entre algunos temas con país. Como se explicó en la metodología, se realizaron pruebas de hipótesis bivariada que nos dan respuesta a este supuesto, las asociaciones significan lo siguiente:

Una relación lineal positiva entre dos variables $X$ e $Y$ significa que los valores de las dos variables varían de forma parecida: los sujetos que puntúan alto en $X$ tienden a puntuar alto en $Y$ y los sujetos que puntúan bajo en $X$, bajo en Y. Una relación lineal negativa significa que los valores de ambas variables cambian justamente al revés: los sujetos que puntúan alto en $X$ tienden a puntuar bajo en $Y$ y los sujetos que puntúan bajo en $X$ tienden a puntuar alto en Y (Pardo y Ruiz 2005, 429).

En el caso panameño, se da una asociación negativa muy baja con el tema de violencia, es decir, a mayor número de noticias sobre violencia, menor la frecuencia de que sean publicaciones de Panamá.

En el caso costarricense ocurre lo mismo, a mayor número de noticias sobre violencia, menor la frecuencia de Costa Rica. Además, existe una asociación muy baja con Salud, Sexualidad y Tecnología.

Para Nicaragua existe asociación estadística muy baja entre Accidentes (tránsito, laborales, hogar, etc.) e Institucionalización. Sobre Guatemala hay una asociación estadística muy baja entre el Mundo del trabajo y con la Participación social.

El Salvador presenta asociación estadística muy baja en Accidentes (tránsito, laborales, hogar, etc.) e institucionalización y una asociación inversa (negativa) muy baja sobre Tecnología, es decir, a mayor número de noticias sobre Tecnología, menor la frecuencia de que sean publicaciones de El Salvador.

Por último, y el caso más significativo es Honduras, donde se presenta la asociación estadística más amplia de todos los datos, asociado con el tema de violencia. Es el país donde es más clara la asociación estadística con Violencia y, además, tiene relaciones negativas en los temas de Deporte y Cultura, Educación y el Mundo del Trabajo, es decir, a mayor cantidad de noticias sobre Deporte y Cultura, Educación y el Mundo del Trabajo menor la frecuencia de que sea Honduras.

Sobre la discusión general y con las asociaciones mostradas, podemos decir que hay mayores representaciones de personas "jóvenes-violencia" en el caso de Honduras y no se representarán así en mayor medida en Costa Rica y Panamá. 
Discusión: del "El tesoro de la patria" a "La escoria" (sic)

Aunque se muestra estadísticamente definitorio, esta relación binaria más claramente en Honduras, de fondo la discusión sigue siendo la misma, el diario El Siglo (2016) publica, en su columna de opinión, el texto "Jóvenes descartables", en el que su escritor presenta la realidad que acaece a la mitad de la población de niños, niñas y las y los jóvenes en Guatemala, pero también insinúa estas dos comprensiones acerca de cómo son pensados los jóvenes, según sus propias palabras, pasando de ser "el tesoro de la patria" a "la escoria" todos aquellos que no tiene un futuro prometedor.

Sobre las personas jóvenes recae esta comprensión, algunos son el mañana próspero y otros ya están demasiado extraviados como para aportar algo. Como se mostró, en la generalidad del material noticioso es frecuente el desdoblamiento para las juventudes, los estudiantes o los sujetos en riesgo, señala Zúñiga $(2010,57)$ que "la institucionalidad social para reproducirse y gobernar, necesita de modelos y monstruos, estos como proyecciones simbólicas para subordinar la justificación a la ley institucional".

Sea por afirmación o negación, su paso, permanencia o expulsión en la familia, la escuela, el barrio, la comunidad, etcétera, y las relaciones de subordinación o conflicto que se establezcan, determinarán las estrategias y actitudes institucionales que se generen en torno a ellos, para cada una de estas esferas institucionales (Pérez 2000).

Como conocimos en la figura de temas (figura 3), la Violencia es el tema que más se menciona a la juventud, por lo que este dato debe ser de atención, y apelar a la juventud como etapa de transición y la asociación ligera de jóvenes con violencia puede enjuiciar a este sector como responsables de esta. Señala Zúñiga:

Cuando las personas jóvenes, son parte de esos procesos de exclusión, son susceptibles de convertirse en monstruos (maldad pura, irracionalidad absoluta, insociabilidad). El monstruo se convertirá en Oveja Negra que intentará ser absorbida para presentarse como un triunfo del sistema, ya sea por derrota o por cooptación (Zúñiga, 2010, 51).

Seguidamente, algunos ejemplos de cómo se presenta esta tendencia en noticias: el diario CR Hoy (Sánchez 2013) titula, en el apartado de sucesos, "Jó- 
venes delincuentes saturan el sistema penitenciario, al punto que seis cárceles ya no reciben detenidos", para esto es consultado el jefe de seguridad de adaptación social de Costa Rica, quien señala que la delincuencia juvenil ha provocado serios problemas de hacinamiento en todas las cárceles del país, afirma: "Es una población complicada, muy violenta. Tenemos el ámbito de adulto joven de La Reforma lleno por eso tenemos gente en la cárcel para menores". Planteado de esta forma, refuerza la asociación joven-violencia, entrando entonces en el ámbito de los jóvenes malos y vinculado con el espacio carcelario.

Otro ejemplo es la publicación de Panamá América (2016), en la que se señala explícitamente la preocupación por los altos niveles de violencia y la cantidad de jóvenes que delinquen, que son fruto de la falta de "ejemplos dignos de imitarse, influencia negativa del entorno, presión de grupo, desorientación infanto-juvenil, crisis del núcleo básico de la sociedad y vacíos existenciales". En este caso, tampoco hay una problematización sobre el delito cometido por las personas y, por el contrario, se responsabiliza de forma absoluta a los individuos, sus vacíos existenciales o la imitación de malos ejemplos.

Sobre este mismo eje, el titular "Asalto con menores aviva el debate sobre la inseguridad ciudadana" (Panamá América 2015, "a") señala que "las autoridades de turno deben tomar medidas urgentes para frenar la criminalidad juvenil, para que lo que hoy es un dolor de cabeza, mañana no sea un cáncer social". Este ejemplo no solo relaciona jóvenes e inseguridad ciudadana, sino que también hace cita de otro adjetivo, como los ya mencionados: "escorias", "cáncer social", "violentos" y "desorientados"9.

Lo preocupante de esta mirada binaria es que tiene una grave consecuencia, no solo porque se consideran como amenaza, sino porque se pasa la página y no se cuestiona entonces por qué los jóvenes quedaron fuera de los roles (espacios, derechos) que la sociedad debió garantizar. Se tratan como iguales el riesgo, el joven malo y el problema social, explica Zúñiga $(2010,63)$ que el periodo neoliberal reveló la necesidad de la política punitiva que defendiera la organización social, una explicación del delito que responsabiliza a la estructura social, es decir, evidenciar los delitos y los malos jóvenes como los responsables y que es una elección que el individuo tomó y que, por lo tanto, merece ser castigado.

\section{Conclusiones}

Sobre lo desarrollado, el material periodístico en efecto es una buena forma de acercarse a las realidades que se hacen noticiosas; en términos operativos, es una fuente accesible para estar en constante construcción y análisis.

Como era lo pretendido, se calificó a las personas jóvenes en procesos sociales y espacios sociales, como respuesta al momento histórico de la reali-
9. Si retomamos la premisa de que la prensa configura conocimiento, las palabras utilizadas por esta formarán parte del imaginario sobre determinado fenómeno, Aliaga y Escobar (2006) indican: En torno al concepto de imaginario social, "el adjetivo social delimita una acepción más restringida al designar dos aspectos de la actividad

imaginante. Por un lado, la orientación de esta hacia lo social, es decir, la producción de representaciones globales de la sociedad y de todo aquello que se relaciona con ella, por ejemplo, de 'orden social', de los actores sociales y de sus relaciones recíprocas (jerarquía, dominación,

conflicto, etc.)" (Bronislaw

Baczko 1991, citado por Aliaga y Escobar, 27). 
dad centroamericana; ubicar buena parte de las noticias sobre jóvenes en el ámbito de violencia es consecuente en un territorio que ha atravesado conflictos armados, procesos de paz y democratización, pobreza, exclusión y crimen organizado, en que lamentablemente los protagonistas de la violencia son, en buena parte, las personas en edad joven. Esto quedó estadísticamente demostrado en el caso hondureño.

Sin duda que el otro ámbito relevante, como la educación, es invariable por todas las atribuciones individuales y sociales que tiene la enseñanza en cada momento histórico, desde las preocupaciones de los clásicos (Pérez 2008) por la función ideológica y ya en el contexto centroamericano, la educación es relevante en su aporte a la movilidad social, la democratización, la lucha contra la pobreza entre otros alcances, por lo que estar dentro o fuera de este ámbito social se vuelve trascendental.

Lo objetivo en este caso es que en Centroamérica hay una disputa, por la permanencia en la escuela, por el empleo, por la salud, el consumo, etcétera y habrá quienes puedan obtener estos capitales y quienes no, ante esto la lógica binaria cobra sentido.

Dos elementos son importantes de mencionar, el primero es que hay espacios sociales en los que no se menciona a las personas jóvenes, no porque no formen parte sino porque la prensa no los muestra y segundo, en lo compilado solo está la voz de los medios, la mayoría de contenidos están ausentes de la postura de los y las jóvenes.

Este estudio insinúa otros trabajos posibles sobre los medios, sería interesante contrastar con materiales noticiosos de otra naturaleza, como diarios o semanarios universitarios, o con diferentes perspectivas políticas en cada uno de los países. El caso de Honduras merece profundizarse, ya que, si hay una asociación más evidente de la población joven con violencia y asociación negativa con Deporte y Cultura, Educación y el Mundo del Trabajo. De forma hipotética se puede agrupar países, por ejemplo, triángulo norte (El Salvador, Honduras y Guatemala) versus Nicaragua, Costa Rica y Panamá y ver si persiste asociación con violencia en el triángulo o agrupar temas desde la comprensión binaria (buenos o malos) y ver su reproducción por países o grupos de países.

\section{Bibliografía}

Aliaga Sáez, Felipe, y Gerardo Escobar Monje. 2006. «El imaginario social del joven en Chile. Una aproximación teórica al concepto del joven problema». Aposta. Revista de Ciencias Sociales (31): 1-17

Barrantes, Alberto. 2013, marzo 21. «Jóvenes ninis vagan entre falta de oportunidades y desinterés» La Nación. 
https://www.nacion.com/archivo/jovenes-ninis-vagan-entre-falta-deoportunidades-y-desinteres/CRXR3G4ZLBA3DDKAMDJ5E3CXHI/storyl Borunda Escobedo, José Eduardo; (2013). «Juventud lapidada: el caso de los ninis.» Nóesis. Revista de Ciencias Sociales y Humanidades, 120-143. Acceso el 27 de agosto de 2019.http://www.redalyc.org/articulo.oa?id=85927875006

Centeno Orozco, Rebeca. 2014. «Discursos públicos y construcción de ciudadanía: ¿poder de los medios de comunicación?». Orbis. Revista Científica Ciencias Humanas 10 (28): 120-133

CR Hoy. 2013, junio 26. «Aumenta el consumo de marihuana entre jóvenes costarricenses y baja el de tabaco» CR Hoy, Edición digital. http://www.crhoy.com/archivo/aumenta-el-consumo-de-marihuanaentre-jovenes-costarricenses-y-baja-el-de-tabaco/nacionales/

CR Hoy. 2013, junio 26. «Aumenta el consumo de marihuana entre jóvenes costarricenses y baja el de tabaco» CR Hoy, Edición digital. http://www.crhoy.com/archivo/aumenta-el-consumo-de-marihuanaentre-jovenes-costarricenses-y-baja-el-de-tabaco/nacionales/

Duarte Quapper, Klaudio, y Catalina Littin Menz. 2002. Niñas, Niños y Jóvenes: Construyendo imágenes en la Prensa escrita. Santiago-Chile: Asociación Chilena pro Naciones Unidas, 2002.

El Siglo. 2016, mayo 12. «Desnutrición y violencia amenazan a niños y jóvenes» El Siglo, Edición digital. http://s21.gt/2016/06/12/desnutricionviolencia-amenazan-a-ninos-jovenes/

Estado de la Región. 2013. Quinto informe de Estado de la Región. Acciones colectivas en Centroamérica. Estado de la Región. Acceso el 29 de mayo de 2018. http://www.estadonacion.or.cr/files/estadisticas/centroamerica/base-dedatos/2013/Manual\%20Acciones\%20Colectivas2013.pdf

Ferrigno, Victor. 2016, mayo 23. «Niños y jóvenes descartables» El Siglo, Edición digital. http://s21.gt/2016/05/23/ninos-jovenes-descartables/

Figueroa Ibarra, Carlos. 2011. «Centroamérica, Neoliberalismo y violencia. Los retos centroamericanos en el siglo XX|» Acta Científica XXIX 
Congreso de la Asociación Latinoamericana de Sociología 2013. Mesa N1 Violencia y sociedad en Centroamérica. http://actacientifica.servicioit.cl/biblioteca/pn/PN7/P_Figueroa \%20lbarra.pdf

Guerrero, Angie. 2013, diciembre 1. «Golpear con el puño cerrado se convierte en juego mortal entre jóvenes» CR Hoy, Edición digital. http:// www.crhoy.com/archivo/portada-golpear-con-el-puno-cerrado-seconvierte-en-juego-mortal-entre-jovenes/tendencias/

Jiménez, Everardo. 2016. «La violencia en el Triángulo Norte de Centroamérica: una realidad que genera desplazamiento». Papel Político 21(1): 167-196

Leitón, Patricia. 2013, marzo 17. «300.000 jóvenes del país trabajan o buscan trabajo» La Nación. https://www.nacion.com/archivo/300-000jovenes-del-pais-trabajan-o-buscan-trabajo/ Q6Y3HD2J75G2NCHZK554UONJWM/storyl

Lemus, Leslie. 2018. «Guatemala: repensando el vínculo entre juventud y violencia en la posguerra». LiminaR. Estudios Sociales y Humanísticos 16(2): 45-59.

Mayorga Rojel, Alberto, Carlos del Valle Rojas. 2008. «Análisis de la configuración semiótica de Chile y Perú en la prensa escrita». Quórum Académico 5(1): 17-40.

Mejía Navarrete, Julio. 2005. «Medios de comunicación y violencia. Los Jóvenes Pandilleros de Lima». Espacio Abierto 14 (3): 389-404.

Menéndez Eduardo L., Di Pardo Renée B. 2008. «La representación social negativa de los procesos de salud/enfermedad/atención en la prensa escrita». Salud colectiva 4(1): 9-30. Acceso el 27 de agosto de 2019. http://www.scielo.org.ar/scielo.php?script=sci_arttext\&pid=S1851$82652008000100002 \& \operatorname{lng}=e s$.

Novo, Yariela. 2013, julio 20. «Jóvenes aplican peligrosas técnicas para seguir la moda de tener las "piernas separadas"» CR Hoy, Edición digital. http://www.crhoy.com/archivo/jovenes-aplican-peligrosastecnicas-para-seguir-la-moda-de-tener-las-piernas-separada/tecnologia/ 
Núñez, Pedro. 2015. Las juventudes disputadas. Aportes para un campo en construcción. Compilado por Diego Beretta. Rosario: Editorial de la Universidad del Rosario.

Panamá América. 2015, febrero 21, "c". «Empresa crea "clones" de mascotas en peluche» Panamá América, Edición digital. http://www.panamaamerica.com.pa/interesante/empresa-crea-clonesde-mascotas-en-peluche-965113

Panamá América. 2014, septiembre 14. «Delincuencia aumenta porque jóvenes no encuentran empleo, según experto» Panamá América, Edición digital. http://www.panamaamerica.com.pa/nacion/delincuenciaaumenta-porque-jovenes-no-encuentran-empleo-segun-experto

Panamá América. 2015, agosto 24, "a". «Asalto con menores aviva el debate sobre la inseguridad ciudadana» Panamá América, Edición digital.http:// www.panamaamerica.com.pa/nacion/asalto-con-menores-aviva-eldebate-sobre-la-inseguridad-ciudadana-989409

Panamá América. 2015, diciembre 17, "b". «Gala de jóvenes sobresalientes» Panamá América. http://www.panamaamerica.com.pa/gente-con-estilo/ gala-de-jovenes-sobresalientes-1005190

Panamá América. 2016, agosto 08. «Tu aporte ayuda a niños y jóvenes» Panamá América, Edición digital. https://www.panamaamerica.com.pa/ey/tu-aporte-ayuda-ninos-yjovenes-1037852

Pardo, A. y Ruiz, M. 2005. Análisis de datos con SPSS 13 Base. Madrid: McGraw-Hill, 2005.

Pérez Islas, José Antonio. 2008. Teorías sobre la juventud. Las miradas de los clásicos. Coordinado por José Antonio Pérez Islas, Mónica Valdez González y María Herlinda Suárez Zozaya. México D. F.: Porrúa.

Pérez, José Antonio. (2000). «Ser Joven en México: conceptos y contextos». Educación y Ciudadanía A. C. Acceso el 27 de agosto de 2019. http://www.educiac.org.mx/pdf/Biblioteca/Juventud_e_Identidad/019Ser Joven_en_Mexico.pdf 
Reguillo, Rossana (2000). Emergencias de culturas juveniles. Enciclopedia Latinoamericana de Sociocultura y Comunicación. Bogotá, Colombia: Editorial Norma.

Rodríguez, Ernesto. 2000. «Juventud y desarrollo en América Latina: desafíos y prioridades en el comienzo de un nuevo siglo» Políticas públicas de juventud en América Latina: desafíos y prioridades a comienzos de un nuevo siglo, Seminario La Renovación del Capital Humano y Social: la Importancia Estratégica de Invertir en el Desarrollo y la Participación de los Jóvenes. Cuadragésima Primera Reunión Anual de la Asamblea de Gobernadores del Banco Interamericano de Desarrollo, Nueva Orleans, 24 al 27 de marzo de 2000. http://ibero.mx/campus/publicaciones/jovenes/pdf/epieck2.pdf

Rodríguez, Ernesto. 2003. «Políticas públicas de juventud en América Latina: de la construcción de espacios específicos, al desarrollo de una perspectiva generacional». Revista Latinoamericana de Ciencias Sociales, Niñez y Juventud 1(2).

Rojas, Pablo. 2013, octubre 30. «Ticos con más problemas de empleo en Centroamérica; recomiendan medidas para encontrar trabajo» CR Hoy, Edición digital. http://www.crhoy.com/archivo/jovenes-ticos-con-masproblemas-de-empleo-en-centroamerica-recomiendan-medidas-paraencontrar-trabajo/nacionales/

Ross, Amy. 2013, octubre 28. «Fiestas exponen mucho a los jóvenes a delitos $y$ amenazas» La Nación. https://www.nacion.com/el-pais/educacion/fiestas-exponen-mucho-alos-jovenes-a-delitos-y-amenazas/ WPC2PBGHPBFQVNXCCZOKCBOWCI/story/

Ruiz, Anaed (2016, 10, 14) «Se estima que solo hay 35 mil puestos de trabajo» El Siglo. http://s21.gt/2016/10/14/35-mil-puestos/

Saintout, Florencia. 2013. Los jóvenes en la Argentina: Desde una epistemología de la esperanza. Buenos Aires: Universidad Nacional de Quilmes Editorial, 2013. 
Salama, Pierre. 2008. «Informe sobre la violencia en América Latina». Revista de Economía Institucional 10 (18): 81-102

Sánchez, Álvaro. 2013, mayo 6. «Jóvenes delincuentes saturan el sistema penitenciario, al punto que seis cárceles ya no reciben detenidos» $C R$ Hoy, Edición digital. http://www.crhoy.com/archivo/jovenes-delincuentessaturan-el-sistema-penitenciario-al-punto-que-seis-carceles-ya-noreciben-detenidos/nacionales/

Vargas, Monserrath. 2015, julio 5. «Jóvenes 'del milenio' en Costa Rica persiguen su felicidad antes del dinero» La Nación. https://www.nacion.com/ciencia/aplicaciones-cientificas/jovenes-delmilenio-en-costa-rica-persiguen-su-felicidad-antes-del-dinero/ 6RPD3EN435ECHJ5P6YNETEHQMY/storyl

Vega Jiménez, Patricia. 2016. «La investigación sobre Comunicación en Centroamérica (1980-2015) ». Anuario Electrónico de Estudios en Comunicación Social "Disertaciones" 9 (2): 117-138

Waiselfisz, Juan Jacobo (2008). «Mapa de la violencia: los jóvenes de América Latina 2008» http://www.mapadaviolencia.org.br/publicacoes/ Mapa_2008_al.pdf

Zúñiga Núñez, Mario. 2010. Pensar a los jóvenes: Más allá de modelos o monstruos: San José, Costa Rica: Departamento Ecuménico de Investigación DEI. 